\title{
INFINITE PRODUCTS AND PARACONTRACTING MATRICES *
}

\author{
W.-J. BEYN ${ }^{\dagger}$ AND L. ELSNER ${ }^{\dagger}$
}

\begin{abstract}
In [Linear Algebra Appl., 161:227-263, 1992] the LCP-property of a finite set $\Sigma$ of square complex matrices was introduced and studied. A set $\Sigma$ is an LCP-set if all left infinite products formed from matrices in $\Sigma$ are convergent. It was shown earlier in [Linear Algebra Appl., 130:65-82, 1990] that a set $\Sigma$ paracontracting with respect to a fixed norm is an LCP-set. Here a converse statement is proved: If $\Sigma$ is an LCP-set with a continuous limit function then there exists a norm such that all matrices in $\Sigma$ are paracontracting with respect to this norm. In addition the stronger property of $\ell$-paracontractivity is introduced. It is shown that common $\ell$-paracontractivity of a set of matrices has a simple characterization. It turns out that in the above mentioned converse statement the norm can be chosen such that all matrices are $\ell$-paracontracting. It is shown that for $\Sigma$ consisting of two projectors the LCP-property is equivalent to $\ell$-paracontractivity, even without requiring continuity.
\end{abstract}

AMS(MOS) subject classification. 65F 10, 47H09, 15 A99

Key words. Convergence, infinite products, LCP-property, product boundedness, paracontracting matrices, norms, projections

1. Introduction. In the investigation of chaotic iteration procedures for consistent linear systems, matrices which are paracontracting with respect to some vector norm play an important role. It was shown in [3], that if $A_{1}, \ldots, A_{m}$ are finitely many $k \times k$ complex matrices which are paracontracting with respect to the same norm, then for any sequence $d_{i}, 1 \leq d_{i} \leq m, i=$ $1,2, \ldots$ and any $x_{0}$ the sequence

$$
x_{i}=A_{d_{i}} x_{i-1} \quad i=1,2, \ldots
$$

is convergent. In particular $A^{(d)}=\lim _{i \rightarrow \infty} A_{d_{i}} \ldots A_{d_{1}}$ exists for all sequences $\left\{d_{i}\right\}_{i=1}^{\infty}=d$. Hence those sets are examples of sets of matrices all infinite products of which converge. Such sets have been studied in [2]. Following [2], we call them LCP-sets.

In this note we investigate the question of necessity. As our main result we show that under the additional assumption that the mapping

$$
d=\left\{d_{i}\right\}_{i=1}^{\infty} \rightarrow A^{(d)}=\lim _{i \rightarrow \infty} A_{d_{i}} A_{d_{i-1}} \ldots A_{d_{1}}
$$

\footnotetext{
* Received by the editors on 8 October 1996. Final manuscript accepted on 26 February 1997. Handling editor: Daniel Hershkowitz.

$\dagger$ Fakultät für Mathematik, Universität Bielefeld, Postfach 100131, D-33501 Bielefeld, Germany (beyn@mathematik.uni-bielefeld.de, elsner@mathematik.uni-bielefeld.de).

$\ddagger$ This paper was partially written during a stay at the University of Calgary. This author thanks the members of the Department of Mathematics, in particular P. Lancaster and P. Binding, for their hospitality and support.
} 
is continuous (which is equivalent to the set of fixed points of $A_{i}$ being the same for all $1 \leq i \leq m)$, an $\mathrm{LCP}$-set is necessarily paracontracting with respect to some norm. In this sense paracontractivity is equivalent to the LCP-property. We show, in addition, that continuity implies even the stronger property of $\ell$-paracontractiveness.

In the final section, we consider the case $m=2$. We show that for $\Sigma$ consisting of two projectors the LCP-property is equivalent to $\ell$-paracontractivity, even without continuity.

2. Notations and known results. Let \|\| denote a vector norm in $\mathbb{C}^{k}$. A $k \times k$ matrix $P$ is paracontracting with respect to \|\| , if for all $x$

$$
P x \neq x \Leftrightarrow\|P x\|<\|x\| .
$$

We denote by $\mathcal{N}(\|\|)$ the set of all $k \times k$ matrices paracontracting w.r.t. \|\| . We call $P$ l-paracontracting w.r.t. \|\| , if there exists $\gamma>0$ such that

$$
\|P x\| \leq\|x\| \Leftrightarrow \gamma\|P x \Leftrightarrow x\|
$$

holds for all $x \in \mathbb{C}^{k}$ and denote this set of matrices by $\mathcal{N}_{\gamma}(\|\|)$. Obviously

$$
\mathcal{N}_{\gamma}(\|\|) \subset \mathcal{N}(\|\|) .
$$

The example of an orthogonal projection $P, P \neq I, P \neq 0$ which is paracontracting w.r.t. the Euclidean vector norm but never $\ell$-paracontracting shows that in (3) equality does not hold in general.

For a bounded set $\Sigma=\Sigma_{1}$ of complex $k \times k$ - matrices define $\Sigma_{0}=\{I\}$ and for $n \geq 1, \Sigma_{n}=\left\{M_{1} M_{2} \ldots M_{n}: M_{i} \in \Sigma\right\}$, the set of all products of matrices in $\Sigma$ of length $n$. Let $\Sigma=\left\{A_{1}, \ldots, A_{m}\right\}$ be finite. For $d=\left(d_{1}, d_{2}, \ldots\right) \in$ $\{1, \ldots, m\}^{\mathbb{N}}$, i.e. $1 \leq d_{i} \leq m$ for $i \in \mathbb{N}$ define $A^{(d)}=\lim _{n \rightarrow \infty} A_{d_{n}} A_{d_{n-1}} \ldots A_{d_{1}}$, if the limit exists. The set $\Sigma$ is an LCP-set (left-convergent-product), if for all $d \in\{1, \ldots, m\}^{\mathbb{N}}$ the limit $A^{(d)}$ exists. The function $d \rightarrow A^{(d)}$ mapping $\{1, \ldots, m\}^{\mathbb{N}}$ into the space of $k \times k$ - matrices is called the limit function.

We note in passing that in [2] also the right-convergent-product property (RCP) was introduced. For convenience we restrict our considerations to the left convergence case.

Introducing in $\{1, \ldots, m\}^{\mathbb{N}}$ the metric

$$
\operatorname{dist}\left(d, d^{\prime}\right)=m^{-r} \quad r \text { smallest index such that } d_{r} \neq d_{r}^{\prime},
$$

we define the concept of a continuous limit function in the standard way.

The set $\Sigma$ is product bounded, if there exists $\Delta>0$ such that

$$
\|A\| \leq \Delta \quad \text { for all } A \in \Sigma_{n}, n=1,2, \ldots
$$


Here || || denotes any matrix norm. Obviously this concept is independent of the norm. G. Schechtman has proved that LCP-sets are product bounded (see [1, Theorem I]). We have the following result.

LEMMA 2.1. For a set $\Sigma$ of $k \times k$-matrices the following are equivalent.

(i) The set $\Sigma$ is product bounded.

(ii) There exists a vector norm \|\| such that $\|A x\| \leq\|x\|$ for all $A \in \Sigma, x \in$ $\mathbb{C}^{k}$.

(iii) There exists a multiplicative matrix norm \|\| such that $\|A\| \leq 1$ for all $A \in \Sigma$.

Proof. As (ii) $\Longrightarrow$ (iii) (the operator norm is multiplicative) and (iii) $\Longrightarrow$ (i) are obvious, only $(i) \Longrightarrow(i i)$ has to be shown.

For some vector norm $\nu$ define the norm

$$
\|x\|=\sup _{n \geq 0}\left\{\sup _{A \in \Sigma_{n}} \nu(A x)\right\}
$$

which is finite by $(i)$. Then $\|A x\| \leq\|x\|$ for all $A \in \Sigma$. $\square$

We remark that this result could also be derived from [5]. For a given matrix norm \|\| and bounded $\Sigma$ let $\hat{\rho}_{n}=\hat{\rho}_{n}(\Sigma)=\max \left\{\|A\|, A \in \Sigma_{n}\right\}$ and let $\hat{\rho}=\hat{\rho}(\Sigma)=\lim _{n \rightarrow \infty} \hat{\rho}_{n}^{1 / n}$. The quantity $\hat{\rho}$ is called the joint spectral radius of $\Sigma$. It was introduced in [5] for general bounded sets in a normed algebra. In [5] and in [2] the limit is replaced by lim sup, however, it is implicitly shown in [2] (see there (3.12)), that the limit exists.

We give here a characterization of $\hat{\rho}(\Sigma)$, which can be found essentially in [5]. Hence the proof, which is also an easy consequence of the previous Lemma, is omitted.

LEMMA 2.2. For any bounded set $\Sigma$ of $k \times k$ - matrices

$$
\widehat{\rho}(\Sigma)=\inf _{\nu \text { operator norm }} \sup _{A \in \Sigma} \nu(A)
$$

The following result is just a restatement of the Theorem in [3].

THEOREM 2.3. Let $\Sigma \subset \mathcal{N}(\|\|)$ for some vector norm \|\| , $\Sigma$ finite. Then $\Sigma$ has the LCP-property.

We finish this section by pointing out that if in addition $\Sigma \subset \mathcal{N}_{\gamma}(\|\|)$ for some positive $\gamma$, then the proof of Theorem 2.3 is very simple. This is outlined below. It is a consequence of the following characterization of $\ell$ paracontractivity of the set $\Sigma$.

Let $\Sigma=\left\{A_{i}\right\}_{i \in I}$ be a set of matrices, not necessarily finite. Let $d=$ $\left(d_{1}, \ldots, d_{r}\right) \in I^{r}, \nu$ a vector norm. Define

$$
\nu_{d}(x)=\nu\left(x_{r}\right)+\sum_{k=1}^{r} \nu\left(x_{k} \Leftrightarrow x_{k-1}\right)
$$


where the vectors $x_{i}$ are defined as in (1) and $x=x_{0}$. Then obviously, for any $i \in I$ and $d^{\prime}=\left(i, d_{1}, \ldots, d_{r}\right)$

$$
\nu_{d}\left(A_{i} x\right)=\nu_{d^{\prime}}(x) \Leftrightarrow \nu\left(A_{i} x \Leftrightarrow x\right) .
$$

We define now

$$
\nu_{*}(x)=\sup \left\{\nu_{d}(x): d \text { finite }\right\} .
$$

This is a vector norm provided that $\nu_{\star}(x)<\infty$ for all $x$.

THEOREM 2.4. For a set of $k \times k$-matrices $\left\{A_{i}\right\}_{i \in I}$ the following are equivalent.

(i) There exists a norm $\nu$ and a positive $\gamma$ such that

$$
A_{i} \in \mathcal{N}_{\gamma}(\nu) \quad \text { for all } \quad i \in I .
$$

(ii) There exists a vector norm $\mu$ such that

$$
\mu_{*}(x)<\infty \quad \text { for all } \quad x \in \mathbb{C}^{k}
$$

(iii) For all vector norms $\mu$

$$
\mu_{*}(x)<\infty \quad \text { for all } \quad x \in \mathbb{C}^{k}
$$

Proof. We show $($ i $) \Rightarrow($ iii $) \Rightarrow($ ii $) \Rightarrow(i)$. Assume that $(i)$ holds. Then from

$$
\nu\left(A_{i} x \Leftrightarrow x\right) \leq \gamma^{-1}\left\{\nu(x) \Leftrightarrow \nu\left(A_{i} x\right)\right\} \quad \forall i \in I, \forall x
$$

we have, using the notation in (5), and assuming (w.l.o.g.) that $\gamma \leq 1$,

$$
\begin{aligned}
\nu_{d}(x) & \leq \nu\left(x_{r}\right)+\gamma^{-1} \sum_{k=1}^{r}\left(\nu\left(x_{k-1}\right) \Leftrightarrow \nu\left(x_{k}\right)\right) \\
& =\nu\left(x_{r}\right)+\gamma^{-1}\left\{\nu(x) \Leftrightarrow \nu\left(x_{r}\right)\right\} \leq \gamma^{-1} \nu(x) .
\end{aligned}
$$

If $\mu$ is a fixed vector norm, then due to the compatibility of any two norms we have a constant $\kappa$ such that $\mu(x) \leq \kappa \nu(x)$ and hence also $\mu_{d}(x) \leq \kappa \nu_{d}(x)$. The inequality $(9)$ gives that $\mu_{*}(x)$ exists, hence we have $(i i i)$.

Obviously (iii) implies (ii).

Now we assume (ii). From (6) we have

$$
\mu_{*}\left(A_{i} x\right) \leq \mu_{*}(x) \Leftrightarrow \mu\left(A_{i} x \Leftrightarrow x\right) \leq \mu_{*}(x) \Leftrightarrow \gamma \mu_{*}\left(A_{i} x \Leftrightarrow x\right)
$$

where we have chosen $\gamma$ such that $\mu(\xi) \geq \gamma \mu_{*}(\xi)$ for all $\xi$. Hence $(i)$ holds with $\nu=\mu_{*}$.

We indicate now the easy proof of the fact that a finite set $\Sigma=$ $\left\{A_{1}, \ldots, A_{m}\right\} \subset \mathcal{N}_{\gamma}(\nu)$ has the LCP-property. It suffices to show that for any $x_{0}$ and any $d=\left(d_{1}, d_{2}, \ldots\right) \in\{1, \ldots, m\}^{\mathbb{N}}$ the sequence $\left\{x_{i}\right\}_{i=1}^{\infty}$ defined by (1) is convergent. By Theorem 2.4 we have $\nu_{*}\left(x_{0}\right)<\infty$, hence the sequence $\sum_{i=1}^{\infty} \nu\left(x_{i} \Leftrightarrow x_{i-1}\right)$ is convergent. This implies that the sequence of the $x_{i}^{\prime} s$ is a Cauchy sequence. 
3. Main result. It is tempting to conjecture that the converse statement of Theorem 2.3 also holds, namely that if $\Sigma$ is an LCP-set, then there exists a vector norm \|\| such that $\Sigma \subset \mathcal{N}(\|\|)$. We were unable to decide this question in general. However, the converse is true if $\Sigma$ is an LCP-set with a continuous limit function. More precisely, the following theorem holds.

ThEOREM 3.1. Let $\Sigma=\left\{A_{1}, \ldots, A_{m}\right\}$ be a finite set of $k \times k$-matrices and let the subspaces $M_{i}=N\left(I \Leftrightarrow A_{i}\right), i=1, \ldots, m$. Then the following are equivalent.

(i) The set $\Sigma$ has the $L C P$-property and $M_{i}=M_{j}$ for $i, j=1, \ldots, m$.

(ii) The set $\Sigma$ has the LCP-property with continuous limit function.

(iii) There exists a vector norm \|\| in $\mathbb{C}^{k}$ and a positive $\gamma$ such that $\Sigma \subset$ $\mathcal{N}_{\gamma}(\|\|)$ and $M_{i}=M_{j}$ for $i, j=1, \ldots, m$.

(iv) There exists a vector norm \|\| in $\mathbb{C}^{k}$ such that $\Sigma \subset \mathcal{N}(\|\|)$ and $M_{i}=M_{j}$ for $i, j=1, \ldots, m$.

Proof. We will show $(i) \Longrightarrow($ ii $) \Longrightarrow($ iii $) \Longrightarrow($ iv $) \Longrightarrow(i)$.

To prove $(i) \Longrightarrow(i i)$, we are going to show that

$$
\left\|A^{(d)} \Leftrightarrow A^{\left(d^{\prime}\right)}\right\| \leq(2+\Delta)\left\|A_{(r)} \Leftrightarrow A^{(d)}\right\|,
$$

where \|\| is a fixed operator norm, $(d),\left(d^{\prime}\right) \in\{1, \ldots, m\}^{\mathbb{N}}, d_{i}=d_{i}^{\prime}$ for $i \leq r$, and $\Delta$ is the bound in the definition of product boundedness. Here we use the fact that by [1], $\Sigma$ is product bounded. Also we use the notation

$$
A_{(r)}=A_{d_{r}} A_{d_{r-1}} \ldots A_{d_{1}}, A_{(s)}^{\prime}=A_{d_{s}^{\prime}} \ldots A_{d_{1}^{\prime}} .
$$

Let $M_{0}=N\left(I \Leftrightarrow A_{i}\right), i=1, \ldots, m$ the common pointwise invariant subspace of the matrices $A_{i}$. If $i \in\{1, \ldots, m\}$ occurs infinitely often in the sequence $d_{1}, d_{2}, \ldots$, then by the usual reasoning $A_{i} A^{(d)}=A^{(d)}$, and hence all columns of $A^{(d)}$ are in $M_{0}$. Hence $A_{j} A^{(d)}=A^{(d)}$ for all $A_{j} \in \Sigma$. This implies the relation

$$
A_{(r+s)}^{\prime} \Leftrightarrow A_{(r)}=\left(A_{d_{r+s}^{\prime}} \ldots A_{d_{r+1}^{\prime}} \Leftrightarrow I\right)\left(A_{(r)} \Leftrightarrow A^{(d)}\right) \quad s>0
$$

and hence $\left\|A_{(r+s)}^{\prime} \Leftrightarrow A_{(r)}\right\| \leq(1+\Delta)\left\|A_{(r)} \Leftrightarrow A^{(d)}\right\|$. Taking $s \rightarrow \infty$, we get

$$
\left\|A^{\left(d^{\prime}\right)} \Leftrightarrow A_{(r)}\right\| \leq(1+\Delta)\left\|A_{(r)} \Leftrightarrow A^{(d)}\right\|,
$$

from which (11) follows. This implies continuity: Given $\epsilon>0$, as $A_{(r)} \rightarrow A^{(d)}$, there exists $r_{0}$ such that

$$
\left\|A_{\left(r_{0}\right)} \Leftrightarrow A^{(d)}\right\| \leq(2+\Delta)^{-1} \epsilon .
$$

Now, if $\left(d^{\prime}\right)$ is such that $\operatorname{dist}\left(d, d^{\prime}\right) \leq m^{-r_{0}-1}$, then $d_{i}=d_{i}^{\prime}$ for $i \leq r_{0}$ and hence by (11)

$$
\left\|A^{\left(d^{\prime}\right)} \Leftrightarrow A^{(d)}\right\| \leq(2+\Delta)\left\|A_{\left(r_{0}\right)} \Leftrightarrow A^{(d)}\right\| \leq \epsilon .
$$


We remark that although this step is not directly contained in [2], we have used tools and ideas from that paper.

Finally, we show $($ ii $) \Longrightarrow($ iii). Assume that (ii) holds. By Theorem 4.2 in [2] the subspaces $M_{i}$ are the same for $i=1, \ldots, m$. By a similarity transformation, i.e.

$$
\Sigma \rightarrow S^{-1} \Sigma S=\left\{S^{-1} A_{i} S: i=1, \ldots, m\right\}
$$

which does not change the properties involved, we can assume that $M_{i}$ is spanned by the first $r$ unit vectors $e_{1}, \ldots, e_{r}$, so that for $i=1, \ldots, m$,

$$
A_{i}=\left(\begin{array}{cc}
I_{r} & C_{i} \\
0 & \widetilde{A}_{i}
\end{array}\right)
$$

Obviously $\widetilde{\Sigma}=\left\{\widetilde{A}_{1}, \ldots, \widetilde{A}_{m}\right\}$ has the LCP-property also and its limit function is identically zero. Otherwise if $\tilde{A}^{(d)} \neq 0$, for some $d \in\{1, \ldots, m\}^{\mathbb{N}}$ we would have $\widetilde{A}_{r} \widetilde{A}^{(d)}=\tilde{A}^{(d)}$ for at least one $r$ and $\tilde{A}_{r}$ would have 1 as an eigenvalue. This contradicts our assumptions. But then, from Theorem 4.1 in [2], it follows that $\hat{\rho}(\tilde{\Sigma})<1$. We select some $q$ in $(\hat{\rho}(\tilde{\Sigma}), 1)$. By Lemma 2.2 we find a norm \|\| on $\mathbb{C}^{k-r}$ such that

$$
\left\|\tilde{A}_{i} x\right\| \leq q\|x\| \text { for all } \quad x \in \mathbb{C}^{k-r} \quad \text { and all } i=1, \ldots, m .
$$

Denoting by \|\|$_{2}$ the Euclidean norm in $\mathbb{C}^{r}$, we introduce for any positive $\epsilon$ the following vector norm in $\mathbb{C}^{k}$ :

$$
\mu_{\epsilon}(x)=\mu_{\epsilon}\left(\begin{array}{l}
x_{1} \\
x_{2}
\end{array}\right)=\epsilon\left\|x_{1}\right\|_{2}+\left\|x_{2}\right\| .
$$

Then we observe that

$$
\begin{aligned}
\mu_{\epsilon}\left(A_{i} x\right) & =\mu_{\epsilon}\left(\begin{array}{c}
x_{1}+C_{i} x_{2} \\
\tilde{A}_{i} x_{2}
\end{array}\right) \\
& =\epsilon\left\|x_{1}+C_{i} x_{2}\right\|_{2}+\left\|\tilde{A}_{i} x_{2}\right\| \\
& \leq \epsilon\left\|x_{1}\right\|_{2}+\left(\epsilon\left\|C_{i}\right\|+q\right)\left\|x_{2}\right\|,
\end{aligned}
$$

where $\left\|C_{i}\right\|=\max \left\{\frac{\left\|C_{i} x\right\|_{2}}{\|x\|_{2}}, x \in \mathbb{C}^{k-r}\right\}$. Choose $\epsilon>0$ such that $\tilde{q}=$ $\max _{i}\left(\epsilon\left\|C_{i}\right\|+q\right)<1$ and let $\gamma=(1 \Leftrightarrow \tilde{q}) /(1+\tilde{q})$. Then we get after some manipulations using (12) and (13) the inequality

$$
\mu_{\epsilon}\left(A_{i} x\right) \leq \mu_{\epsilon}(x) \Leftrightarrow \gamma \mu_{\epsilon}\left(A_{i} x \Leftrightarrow x\right) .
$$

Hence $\Sigma \subset \mathcal{N}_{\gamma}\left(\mu_{\epsilon}\right)$ and (iii) is proved.

$($ iii $) \Longrightarrow($ iv $)$ is trivial, while $(i v) \Longrightarrow(i)$ is Theorem 2.3 . 
4. Final remarks. The conjecture at the beginning of the previous section remains unsolved even in the case $m=2$. The following related result was proved in $[6]$.

THEOREM 4.1. For $\Sigma=\left\{A_{1}, A_{2}\right\}$ the following are equivalent.

(i) $\Sigma$ is an LCP-set.

(ii) (a) there exist a vector norm || || such that

$$
\begin{aligned}
& \left\|A_{i} x\right\| \leq\|x\|, \quad i=1,2 \quad \text { for all } \quad x \in \mathbb{C}^{k}, \\
& \left\|A_{1} A_{2} x\right\|=\|x\| \Longrightarrow A_{1} x=A_{2} x=x
\end{aligned}
$$

(b) For $i=1,2$ if $\lambda$ is an eigenvalue of $A_{i},|\lambda|=1$, then $\lambda=1$.

Notice that here we have finitely many conditions characterizing the LCPproperty. Nevertheless (ii) seems not to imply paracontractivity of $\Sigma$.

In the case of two projectors $P_{i}, i=1,2$, not necessarily orthogonal, the conjecture can be proved.

Theorem 4.2. Let $P_{i}, i=1,2$ be projectors, i.e. $P_{i}^{2}=P_{i}, i=1,2$. Then the following are equivalent.

(i) $\left\{P_{1}, P_{2}\right\}$ is an LCP-set.

(ii) There exists a vector norm \|\| and a positive $\gamma$ such that

$$
\left\{P_{1}, P_{2}\right\} \subset \mathcal{N}_{\gamma}(\|\|) \text {. }
$$

The proof is given after the following auxiliary result.

Lemma 4.3. Let $A, B$ be complex $k \times k$-matrices such that

(i) $B$ is convergent, i.e. the powers of $B$ converge, and

(ii) $\lim _{n \rightarrow \infty} A B^{n}=0$.

Then there exists $\alpha \in(0,1)$ such that for any norm \|| \|

$$
\left\|A B^{n}\right\| \leq C \alpha^{n} \quad \text { for all } \quad n \in \mathbb{N} \text {. }
$$

with $C>0$ a constant depending on the norm.

Proof. By eventually changing the basis accordingly, we have by ( $i$ ) that $B$ is of the form

$$
B=\left(\begin{array}{cc}
I_{r} & 0 \\
0 & B_{0}
\end{array}\right)
$$

with $\alpha=\left\|B_{0}\right\|<1$ for a suitable norm. Here $r$ is the dimension of $N(I \Leftrightarrow B)$ and we assume $r>0$. Otherwise nothing has to be proved. Partitioning $A=\left(A_{1}, A_{2}\right)$, where $A_{1}$ contains the first $r$ columns of $A$, we get $A B^{n}=$ $\left(A_{1}, A_{2} B_{0}^{n}\right)$, and we see from (ii) that $A_{1}=0$. But then clearly

$$
\left\|A B^{n}\right\|=\left\|\left(0, A_{2} B_{0}^{n}\right)\right\| \leq C \alpha^{n}
$$

for a suitable $C$. 
Proof of Theorem 4.2. Obviously we need only to show the implication (i) $\Longrightarrow($ ii $)$.

Let \|\| denote a vector norm satisfying $\left\|P_{i} x\right\| \leq\|x\|, i=1,2, x \in \mathbb{C}^{k}$ (See Lemma 2.1, (ii)) and define for $n \geq 0$

$$
\begin{aligned}
a_{n}(x) & =\left\|\left(P_{1} \Leftrightarrow I\right)\left(P_{2} P_{1}\right)^{n} x\right\| \\
b_{n}(x) & =\left\|\left(P_{2} \Leftrightarrow I\right) P_{1}\left(P_{2} P_{1}\right)^{n} x\right\| \\
c_{n}(x) & =\left\|\left(P_{2} \Leftrightarrow I\right)\left(P_{1} P_{2}\right)^{n} x\right\| \\
d_{n}(x) & =\left\|\left(P_{1} \Leftrightarrow I\right) P_{2}\left(P_{1} P_{2}\right)^{n} x\right\|
\end{aligned}
$$

By (i) the sequence

$$
x_{0}=x, x_{2 i+1}=P_{1} x_{2 i}, x_{2 i+2}=P_{2} x_{2 i+1}, i=0, \ldots
$$

is convergent, which gives that $a_{n}(x)=\left\|x_{2 n+1} \Leftrightarrow x_{2 n}\right\| \rightarrow 0$ and $b_{n}(x)=$ $\left\|x_{2 n+2} \Leftrightarrow x_{2 n+1}\right\| \rightarrow 0$. The analogous result holds for $c_{n}$ and $d_{n}$. Similarly we prove that the matrices $P_{1} P_{2}$ and $P_{2} P_{1}$ are convergent. Hence by the previous Lemma $r_{n}(x) \leq C \alpha^{n}$ for suitable $C>0, \alpha \in(0,1)$ and $r=a, b, c, d$. This shows that the following expression

$$
\|x\|_{*}=\|x\|+\max \left(\sum_{n=0}^{\infty}\left(a_{n}(x)+b_{n}(x)\right), \sum_{n=0}^{\infty}\left(c_{n}(x)+d_{n}(x)\right)\right)
$$

is finite, and it is easy to see that $\|x\|_{*}=0$ if and only if $x=0$. Hence it is a norm in $\mathbb{C}^{k}$. (This is essentially the same construction as in $(7)$, but in this special case we can give a closed expression for the norm). By some simple manipulations we get

$$
\left\|P_{1} x\right\|_{*} \leq\|x\|_{*} \Leftrightarrow a_{0}(x)=\|x\|_{*} \Leftrightarrow\left\|P_{1} x \Leftrightarrow x\right\|
$$

and the same result for $P_{2}$. As there is a $\gamma>0$ satisfying $\|x\| \geq \gamma\|x\|_{*}$ we see that $\left\{P_{1}, P_{2}\right\} \subset \mathcal{N}_{\gamma}\left(\|\|_{*}\right)$.

\section{REFERENCES}

[1] Marc A. Berger and Yang Wang. Bounded Semigroups of Matrices. Linear Algebra Appl., $166: 21-27,1992$.

[2] I. Daubechies and J.C. Lagarias. Sets of Matrices All Infinite Products of Which Converge. Linear Algebra Appl., 161:227-263, 1992.

[3] L. Elsner, I. Koltracht and M. Neumann. On the Convergence of Asynchronous Paracontractions with Applications to Tomographic Reconstruction from Incomplete Data. Linear Algebra Appl., 130:65-82, 1990.

[4] S. Nelson and M. Neumann. Generalization of the Projection Method with Applications to SOR Method for Hermitian Positive Semidefinite Linear Systems. Numer. Math., $51: 123-141,1987$.

[5] G.-C. Rota and W.G. Strang. A Note on the Joint Spectral Radius. Indagationes, $22: 379-381,1960$.

[6] L. Elsner and S. Friedland. Norm Conditions for Convergence of infinite products. Linear Algebra Appl., 250:133-142, 1997. 